\title{
Beyond 25 Gb/s Directly-Modulated Widely Tunable VCSEL for Next Generation Access Network
}

\author{
Alberto Gatto ${ }^{1}$, Paola Parolari ${ }^{1}$, Christian Neumeyr $^{2}$ and Pierpaolo Boffi ${ }^{1}$ \\ ${ }^{1}$ Politecnico di Milano, Dip. di Elettronica, Informazione e Bioingegneria, via G. Ponzio 34/5, 20133 Milano, Italy \\ ${ }^{2}$ Vertilas GmbH, Daimlerstr. 11d, D-85748 Garching, Germany \\ Author e-mail address: alberto.gatto@polimi.it
}

\begin{abstract}
We demonstrate capacities beyond $25 \mathrm{~Gb} / \mathrm{s}$ up to $40 \mathrm{~km}$ in the whole C-band range without any dispersion compensation by DMT direct modulation and direct detection exploiting widely tuneable MEMS-VCSELs for future low-cost high-capacity access networks.

OCIS codes: (060.2330) Fiber optics communications; (140.7260) Vertical cavity surface emitting lasers; (060.4250) Networks.
\end{abstract}

\section{Introduction}

The continuous growth of bandwidth demand in the optical access to support different data traffic including mobile backhaul and fronthaul is pushing the research towards a line rate increase beyond $10 \mathrm{~Gb} / \mathrm{s}$. The challenge is to perform this upgrade in a cost-effective way by avoiding expensive premium optical parts [1]. For this reason, in NG-PON2 Full-Service Access Network (FSAN) Group decided to increase the aggregate bitrate using the wavelength dimension in combination with the time dimension, achieving Time and Wavelength Multiplexed-PON (TWDM-PON) [2]. Thus, the next proposals targeting a line rate increase should also be compliant with TWDM-PON standard. In particular, the optical network unit (ONU) transmitters have to be colorless, preferably tunable over 4-8 times the grid-spacing, with very high side-mode suppression (SMSR) to avoid crosstalk into neighboring channels. Moreover, in addition to these stringent requirements for transmitters, to keep the cost low, direct-detection receivers using limited bandwidth optical components should be used to enable $25 \mathrm{G}$ and $40 \mathrm{G}$ PONs.

In this paper, we present for the first time the exploitation of a long-wavelength widely tunable vertical-cavity surface-emitting laser (VCSEL) [3] to achieve a colorless ONU transmitter, presenting low-cost and reduced footprint. Discrete multitone modulation (DMT) combined with direct detection (DD) is employed to use limited-bandwidth VCSELs and standard receivers suitable for $10 \mathrm{~Gb} / \mathrm{s}$ operation, targeting a transported upstream (US) capacity greater than $25 \mathrm{~Gb} / \mathrm{s}$ up to $40-\mathrm{km}$ standard single-mode fiber (SSMF) without any chromatic dispersion (CD) compensation. Transmission up to $20-\mathrm{km}$ SSMF is demonstrated in the NGPON2 US band, while the whole C-band coverage and 40-km SSMF reach are obtained thanks to asymmetrical filtering of the received signals at the central office (CO).

\section{Directly modulated tunable VCSEL}

The employed single-mode widely tunable long-wavelength VCSEL is based on a long wavelength Indium Phosphide (InP) Buried Tunnel Junction (BTJ) VCSEL and features a MEMS top mirror [4]. The small air gap between the surface of the base VCSEL and the MEMS can be thermo-electrically controlled. The change in air gap leads to modehop free tuning of the laser wavelength of about $90 \mathrm{~nm}$ (from $1517 \mathrm{~nm}$ to $1608 \mathrm{~nm}$ ), showing a SMSR above $45 \mathrm{~dB}$ on the entire tuning range. The peak optical fiber-coupled power is $1.2 \mathrm{~mW}$ at $22^{\circ} \mathrm{C}$ with a bias current of $32 \mathrm{~mA}$. The VCSEL has a maximum S-21 3-dB bandwidth of about $7 \mathrm{GHz}$ at $1550 \mathrm{~nm}$, while on a $47-\mathrm{nm}$ wavelength range a minimum bandwidth of $4.5 \mathrm{GHz}$ is guaranteed [5]. Recently, 10-Gb/s directly-modulated non-return-to-zero (NRZ) transmission has been demonstrated for a compensated 40-km SSMF span [6].

The employed VCSEL is packaged in a transmitter optical subassembly (TOSA) with standard LC connector. Inside the TOSA package a thermoelectric cooler, a thermistor and a monitoring diode have been included. Only one control signal is required to tune the laser without mode hops across the full tuning range. In total, only 8 pins are required for the described functionality. Moreover, the exploited device can be assembled inside standardized small form factor pluggable (SFP) optical modules.

\section{Experimental setup}

For the US transmission performance evaluation, we employed the experimental setup shown in Fig. 1. The ONU VCSEL is directly modulated by a DMT signal generated by a Tektronix $50 \mathrm{GS} / \mathrm{s}$ arbitrary-waveform-generator (AWG 70001A) with 14-GHz electrical bandwidth. The DMT signal is calculated by Matlab ${ }^{\circledR}$ and is composed by 255 sub-carriers in $8 \mathrm{GHz}$ range, i.e. the sub-carrier spacing is $31.25 \mathrm{MHz}$. A cyclic prefix $(\mathrm{CP})$ of about $2.1 \%$ of the symbol length is added. The bias current and modulation amplitude are maintained constant at $16 \mathrm{~mA}$ and $950 \mathrm{mV}$, respectively, for the entire $\mathrm{C}$-band wavelength tuning range. 
(C) 2018 Optical Society of America. One print or electronic copy may be made for personal use only. Systematic reproduction and distribution, duplication of any material in this paper for a fee or for commercial purposes, or modifications of the content of this paper are prohibited.

https://doi.org/10.1364/OFC.2018.Th1E.2

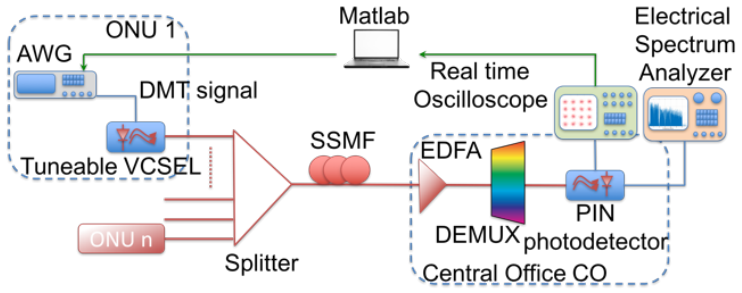

Fig. 1. Experimental setup.

The DMT signal is transmitted over up to 40-km uncompensated SSMF. At the receiver end, a low-noise Erbiumdoped fiber (EDF) preamplifier and a variable optical attenuator (VOA) anticipate a WDM demultiplexer followed by a standard PIN receiver suitable for $10 \mathrm{~Gb} / \mathrm{s}$ operation. The received signal is acquired by a Tektronix real-time oscilloscope (DPO 73304DX) with 8 bits vertical resolution, $50 \mathrm{GS} / \mathrm{s}$ and 33-GHz electrical bandwidth while off-line processing provides digital symbol synchronization, $\mathrm{CP}$ removal, sub-carriers phase recovery and demodulation. The presence at the CO of an EDF preamplifier shared between all the subscribers is compliant with the NGPON2 architecture, nevertheless, the employment of a $10-\mathrm{Gb} / \mathrm{s}$ avalanche photodiode (APD) receiver would allow to avoid the EDF amplifier presence. To cope with the CD accumulated over SSMF propagation distances up to $40 \mathrm{~km}$, we also evaluated the impact of asymmetrical filtering [7], provided by the WDM demultiplexer on the DMT transmission performance. Specifically, in our setup a $0.3-\mathrm{nm}$ tunable optical filter mimics the CO demultiplexer and is fine-tuned with respect to VCSEL emission wavelength thanks to the exploitation of an electrical spectrum analyzer. Performance evaluation is provided comparing results achieved with the filter centered at the VCSEL emission wavelength and with $5 \mathrm{GHz}$ detuning.

\section{Experimental results and discussion}

At first the estimation of the US channel characteristics has been performed by transmitting a probe DMT signal mapped with uniform QPSK loading after direct detection, providing the signal-to-noise ratio (SNR) of each subcarrier. An example of measured SNRs in case of back-to-back (BTB) condition, 40-km SSMF propagation with centered filter and 40-km SSMF propagation with detuned filter is shown in Fig. 2(a) at $1535 \mathrm{~nm}$. After $40 \mathrm{~km}$ of SSMF propagation (red curve), the cumulated chromatic dispersion leads to an evident frequency dip around $5 \mathrm{GHz}$, where the corresponding SNR shows a minimum of about $4 \mathrm{~dB}$. Thanks to the filter detuning (green curve), the impact of chromatic dispersion is mitigated leading to an almost unchanged SNR response with respect to the BTB condition.

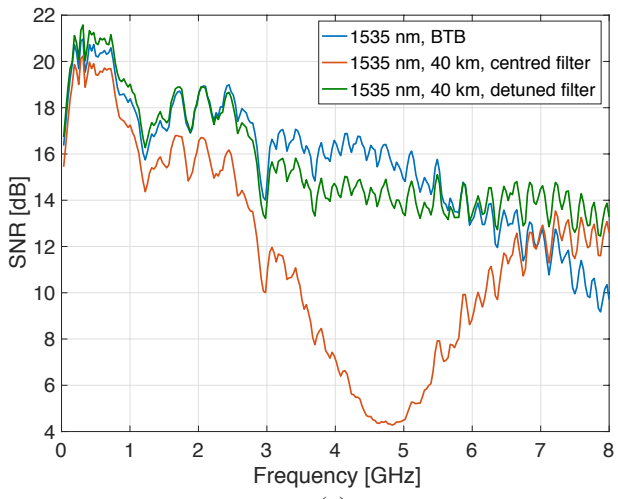

(a)
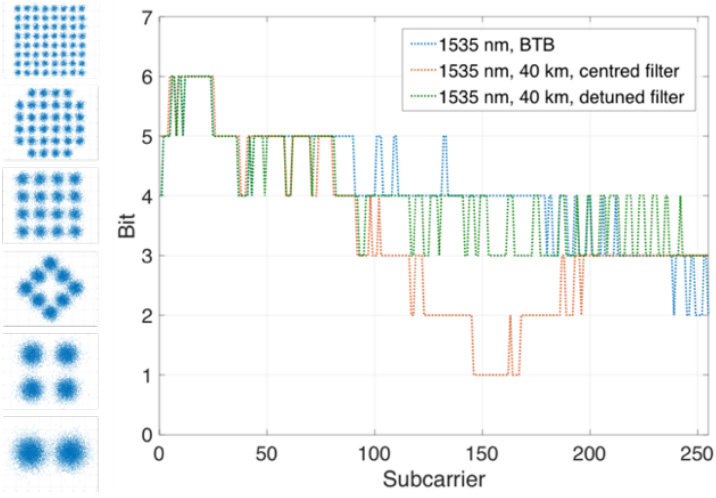

(b)

Fig. 2. (a) Measured SNRs at $1535 \mathrm{~nm}$ and (b) corresponding bit mapping of the 255 sub-carriers for back-to-back condition (blue curve), after 40-km SSMF propagation with centered filter (red curve) and detuned filter (green curve). Examples of the received constellations are shown on the left inset of (b).

The measured SNRs are exploited for performing Chow's algorithm, which is a relatively simple bit- and powerloading procedure for obtaining a good approximation of the highest bit rate achievable by the transmission system [8], setting the target bit error rate (BER) at $3.8 \cdot 10^{-3}$ (useful for exploiting an advanced hard-decision forward error correction code with $7 \%$ overhead). The obtained bits per symbol distribution among the different sub-carriers is reported in Fig. 2(b); in the left side inset, examples of the corresponding received constellations are also displayed.

As expected, a clear improvement of the total capacity transmitted after $40 \mathrm{~km}$ of SSMF is obtained by the detuning of the optical filter, which permits to exploit a bit mapping among the sub-carriers similar to the BTB 
(C) 2018 Optical Society of America. One print or electronic copy may be made for personal use only. Systematic reproduction and distribution, duplication of any material in this paper for a fee or for commercial purposes, or modifications of the content of this paper are prohibited.

https://doi.org/10.1364/OFC.2018.Th1E.2

condition. On the other hand, when the centered filter is exploited, only modulations with lower orders can be achieved, in particular, around the frequency dip at $5 \mathrm{GHz}$, leading to a reduction in the total transported capacity.

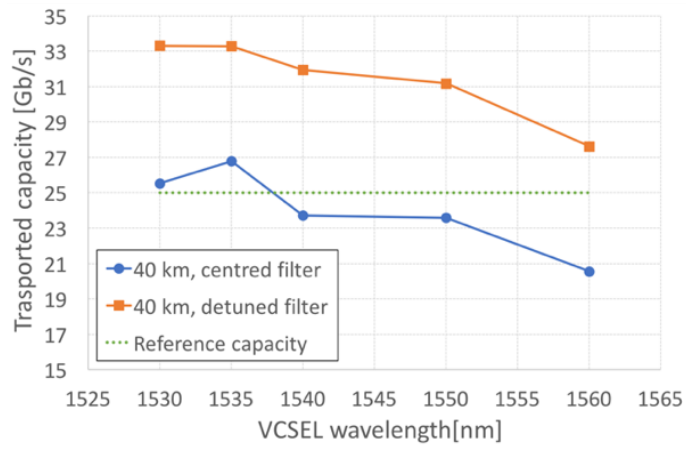

(a)

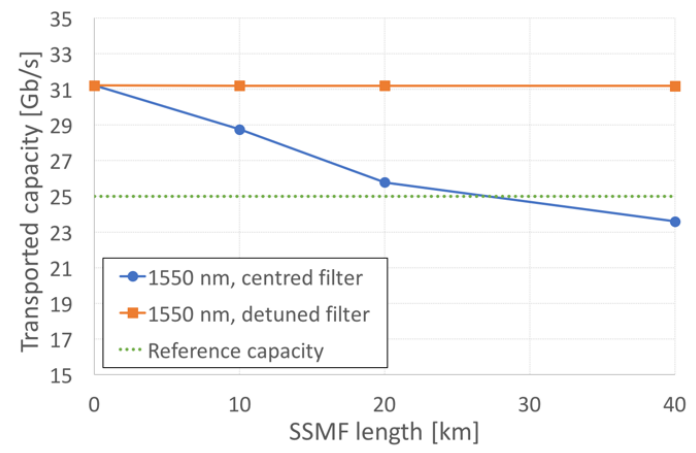

(b)

Fig. 3. (a) Total capacity transported by the system with centered (blue line, circles) and detuned (orange line, squares) filter. (b) Total capacity transported at $1550 \mathrm{~nm}$ vs propagation length in case of centered filter (blue line, circles) and detuned filter (orange line, squares). The reference capacity of $25 \mathrm{~Gb} / \mathrm{s}$ is the green dotted line.

In Fig. 3(a) the total capacity achievable in case of centered (blue line) and detuned (red line) filter is shown for $-6 \mathrm{dBm}$ received optical power, while the green dotted line represents the reference capacity of $25 \mathrm{~Gb} / \mathrm{s}$. In case of filter detuning, in the entire C-band US capacity higher than $27 \mathrm{~Gb} / \mathrm{s}$ are achieved. Since the bias current and the modulation amplitude of the RF drive signal optimized for $1535 \mathrm{~nm}$ are not changed in the test of the whole C-band, a reduction in the transported capacity is noticeable at higher wavelengths, where a different optimization should be performed. On the other hand, when the filter is centered to the VCSEL emission, the transported capacity varies between $27 \mathrm{~Gb} / \mathrm{s}$ at $1535 \mathrm{~nm}$ and $20 \mathrm{~Gb} / \mathrm{s}$ at $1560 \mathrm{~nm}$. Then, the total capacity achievable as function of the SSMF propagation length has been experimentally evaluated. Fig. 3(b) shows the results for $1550 \mathrm{~nm}$ in case of centered (blue line) and detuned (red line) filter with respect to the reference capacity of $25 \mathrm{~Gb} / \mathrm{s}$ (green dotted line). As expected, when the filter is detuned no reduction of the capacity depending on the SSMF length is noticeable, confirming bit-rates higher than $25 \mathrm{~Gb} / \mathrm{s}$ up to $40 \mathrm{~km}$. On the other hand, when the filter is centered, 25-Gb/s capacity is achieved up to $20-\mathrm{km}$ SSMF propagation, which is the minimum reach requested by $10-\mathrm{Gb} / \mathrm{s}$ PONs. In particular, this result is demonstrated for all the wavelengths under $1550 \mathrm{~nm}$.

\section{Conclusions}

DMT US transmission beyond $25 \mathrm{~Gb} / \mathrm{s}$ has been shown by exploiting a widely tunable VCSEL operating over the whole C-band. Uncompensated 20-km SSMF reach is achieved, demonstrating 25-Gb/s capacity in the NGPON2 US band. 40-km SSMF reach can be also obtained thanks to the support of asymmetric filtering. The employment of the experimented tunable VCSEL combined to DMT direct modulation and DD appears as a very promising solution to realized high-bandwidth TWDM PON with low-cost, reduced footprint ONU sources, targeting at least $25 \mathrm{~Gb} / \mathrm{s}$ capacity while exploiting devices suitable for $10 \mathrm{~Gb} / \mathrm{s}$ operation.

This work has been partially supported by the EU PASSION Project (780326).

\section{References}

[1] D. T. van Veen, V. E. Houtsma ,"Proposals for Cost-Effectively Upgrading Passive Optical Networks to a $25 \mathrm{G}$ Line Rate,” J. Lightwave Technol., 35, 1180 (2017).

[2] 40-Gigabit-capable passive optical networks 2 (NG-PON2): Physical media dependent (PMD) layer specification, Recommendation G.989.2, ITU-T, Dec. 2014.

[3] S. Paul et al., "Far-field, linewidth and thermal characteristics of a high-speed 1550-nm MEMS tunable VCSEL", Opt. Expr., Vol. 24, No. 12, 13142-13156 (2016)

[4] S. Paul et al., "High speed surface micromachined MEMS tunable VCSEL for telecom wavelengths," proc. CLEO, AM3K.1, San Jose (2015) [5] S. Paul et al., “10-Gb/s Direct Modulation of Widely Tunable 1550-nm MEMS VCSEL”, IEEE J. Sel. Top. Quant. Electr., Vol. 21, No. 6, p. $1700908(2015)$

[6] C. Wagner et al., "Full C-band Tunable MEMS-VCSEL for Next Generation G.metro Mobile Front- and Backhauling," Proc. OFC, W2A.27, Los Angeles (2017).

[7] A. Dochhan et al., "56 Gb/s DMT Transmission with VCSELs in $1.5 \mathrm{um}$ Wavelength Range over up to $12 \mathrm{~km}$ for DWDM Intra-Data Center Connects", Proc. ECOC, Dusseldorf, Germany (2016).

[8] P. S. Chow et al., "A practical discrete multitone transceiver loading algorithm for data transmission over spectrally shaped channels," IEEE Trans. on comm., Vol. 43, pp. 773-775, (1995). 